\title{
Iris Bünger
}

\section{Apocalypse Now? \\ Kritische Diskursanalyse der Berichterstattung der BILD-Zeitung vom 12.09.01 bis zum 7.11.01}

Nach den Anschlägen vom 11. September überschlug sich die Medienberichterstattung von der ersten Minute an. Die moderne Technik hat es ermöglicht, dass Millionen Menschen weltweit den Zusammensturz der Zwillingstürme des World-Trade-Centers live vor dem Fernseher miterleben konnten. Jede Hollywoodproduktion, die jemals in bezug auf Horror und Schrecken in diese Richtung ging, wurde übertroffen. Die Bilder wurden beständig wiederholt, Augenzeugen, Überlebende, Rettungskräfte, Experten befragt, Reaktionen weltweit aufgenommen, Verdächtige benannt. Kurzzeitig gab es das Bekenntnis einer palästinensischen Splittergruppe, welches bald revidiert wurde. Dann bekannte sich die „Japanische Rote Armee“ zu dem Terroranschlag - als Rache für Hiroshima und Nagasaki. Im Mittelpunkt der Ermittlungen stand von Anfang an - allen Berichten zufolge - jedoch der muslimische Extremist Osama bin Laden, der zurückgezogen in den Bergen Afghanistans lebt, wo er das Gastrecht der Taliban genießt. Er soll über die finanziellen Mittel, die Anhänger und die Infrastruktur verfügen, solche Anschläge planen und durchführen zu können und er soll sie auch angekündigt haben. Die Symbole des Kapitalismus, der Globalisierung, des Wohlstandes und der Macht Amerikas sind getroffen und zerstört. Dies wird in den Medien verstärkt durch die Herz-Symbolik verdeutlicht. Und die Anschläge haben nicht nur Amerika ins „Herz“ getroffen, sondern die gesamte „westliche“ Welt. Noch gibt es keine endgültige Sicherheit über die Identität und Vernetzung derer, die ihr Leben opferten für Heilsversprechen in einer anderen Welt, und es gibt vor allem keine Erkenntnisse darüber, wer noch alles bereit ist, diesen Schritt zu gehen. Und es ist nicht klar, von wo diese Personen - oder Gruppen, Netzwerke - operieren, wie und wo sie moralische Unterstützung finden, um sich und andere zu töten für einen übergeordneten Zweck. Welchen?

Den Medien zufolge verdichteten sich die Beweise gegen den aus SaudiArabien stammenden Osama bin Laden, den Führer der Terrororganisation El Kaida, als Drahtzieher, als Verkörperung der islamistischen Gewalttätigkeit 
und die Hinweise auf seinen Aufenthaltsort in Afghanistan; ein Mensch, der für vergangene Anschläge terroristischer Art verantwortlich oder mitverantwortlich zeichnet; ein Mensch, der die Attentäter beglückwünschte, sich aber nicht schuldig bekannte.

Präsident Georg W. Bush reagierte in den Tagen nach dem Attentat zurückhaltender, als zunächst erwartet. Bush und seine Berater setzten zunächst auf die Bildung einer weltweiten Allianz gegen den Terrorismus. Am 26.9.01 kündigte US-Verteidigungsminister Donald Rumsfeld „einen langen Krieg gegen den Terrorismus“ an unter dem Motto „Operation Dauerhafte Freiheit.“ (FR, 26.9.01, S. 1). Am 02.10.01 wurde dann tatsächlich unter der Angabe, dass Beweise gegen Osama bin Laden vorlägen - die der Öffentlichkeit jedoch nicht präsentiert wurden - der Nato-Bündnisfall erklärt. Am 7.10.01 haben die USA unter Beteiligung Großbritanniens mit Angriffen auf Afghanistan begonnen und bekannt gegeben, der Kampf gegen den Terror sei ,in eine neue Phase getreten." (FR, 8.10.01, S. 1). Am 11.10.01 berichtet die FR auf der Titelseite über Milzbrandfälle, die in den USA zu neuer Angst führen - die steigende Zahl der Betroffenen sind Mitarbeiter von Medienunternehmen. Herkunft und Verbreiter der Viren sind bislang noch unbekannt (FR, 11.10.01). Am 7.12.01 gibt Bundeskanzler Schröder bekannt, dass 3900 Soldaten der Bundeswehr für diesen Krieg zur Verfügung gestellt werden sollen.

Der Krieg wird in Berechtigung, Zielrichtung und Ausprägung kontrovers diskutiert, ob er zur Bekämpfung des Terrorismus beiträgt, ist zumindest zweifelhaft. Es wurde zugegeben, dass versehentlich zivile Ziele u.a. Einrichtungen von Hilfsorganisationen getroffen wurden. Flüchtlingsströme versuchen nicht nur den Bomben, sondern auch dem herannahenden Winter zu entkommen.

Die längerfristige Gefahr, die dieser Krieg, über das unmittelbare menschliche Leid hinaus, mit sich bringt, sollte nicht unterschätzt werden: die Gefahr einer Polarisierung der Welt in „Wir und die Anderen“, in den „Westen und den Rest", in das Christentum und den Islam in einem noch nicht gekannten Ausmaß.

Bei der Herausbildung gesellschaftlicher Wirklichkeit spielen die Medien eine entscheidende Rolle. Im folgenden sollen diskursanalytische Überlegungen zur Berichterstattung der BILD-Zeitung nach dem Anschlag dazu beitragen, Diskursstrategien zu erkennen, die zu einer Polarisierung beitragen. Es wurde eine dezidierte Analyse der Berichterstattung vom 12.9.2001 durchgeführt, da hier die Grundsteine für nachfolgende Diskursstrategie gelegt wurden. In die Analyse werden die weiteren Reportagen in Form einer Strukturanalyse einbezogen, die sich vor allem auf Titel, Schlagzeilen und Anordnung der Berichte bezieht.

Die BILD-Zeitung wurde für diese Analyse gewählt, da sie mit ca. 20 Mio. Lesern als prägend für den Mediendiskurs angesehen wird und zudem ein 
Leitorgan des Journalismus darstellt. Wie Siegfried Jäger bereits in früheren Analysen herausgearbeitet hat, wurde in der BILD-Berichterstattung eine Technik zur Regulierung des Massenbewusstseins entwickelt, die äußerst wirkungsvoll ist. In diesen Analysen konnten anti-demokratische Tendenzen herausgearbeitet werden (vgl. Jäger, S., 1993; Huhnke 1993; Gerhard 1992; van Dijk 1993). Grundlage der folgenden Analyse ist der von Siegfried Jäger entwickelte Ansatz der Kritischen Diskursanalyse (ausführlich beschrieben in Jäger, S., 2001). Bei den folgenden diskursanalytischen Überlegungen kommt der Analyse der Symbolik eine besondere Bedeutung zu - das Verfahren soll hier kurz umrissen werden.

\section{Die Macht der Diskurse ${ }^{1}$}

Diskurs - verstanden als „Fluss von Wissen durch die Zeit“ kommt aus der Vergangenheit, transformiert sich in der Gegenwart und fließt in die Zukunft. „Wissen“ wird in diesem Zusammenhang nicht als Wahrheit verstanden werden, sondern als Für-wahr- oder Für-richtig-Gehaltenes, als alle Arten von Gewusstem, also von Bewusstseinsinhalten insgesamt. Dieser „Fluss von Wissen" wird von miteinander kommunizierenden und kooperierenden Menschen auf der ganzen Welt sehr unterschiedlich aufrechterhalten und mehr oder minder schnell und differenziert verändert. Dennoch haben Diskurse bei allen Unterschieden eine gewisse Festigkeit, eine Struktur. Sie grenzen das Sagbarkeitsfeld ein, d.h. was in einer Gesellschaft zu einer bestimmten Zeit gesagt werden kann, ohne negative Sanktionen nach sich zu ziehen. Sie wirken sich damit auch auf das aus, was getan werden kann. Somit üben Diskurse also Macht aus, was allerdings nicht ausschließt, dass auch Macht über die Diskurse ausgeübt wird. Medien haben u.a. eine solche diskursmächtige Position; sie bilden eine wichtige Instanz, die das Sagbarkeitsfeld vorgibt.

Die Frage wie und wodurch sich Medien und Menschen in den Diskurs verstricken lassen soll nicht auf der Grundlage der traditionellen psychologischen Theoriebildung angegangen werden, sondern anknüpfend an die diskurstheoretischen Überlegungen von Michel Foucault, wie sie in den vergangenen Jahren von Jürgen Link und Siegfried Jäger weiterentwickelt ${ }^{2}$ und in empirischen Untersuchungen des Duisburger Institutes für Sprach- und Sozialforschung (DISS) ausdifferenziert wurden. ${ }^{3}$ Hierbei kommt auch der Tätig-

1 Vgl. zu diesem Abschnitt mit weiterführenden Literaturangaben Jäger, S. (1997).

2 Vergleiche insbesondere Foucault (1977, 1978, 1987) sowie Link (bes. 1982, 1992). Daneben gibt es eine ganze Reihe von Ansätzen, die sich selbst ebenfalls als diskurstheoretisch bezeichnen, die allerdings nicht an Foucault anknüpfen. Diese Ansätze sind anderenorts dargestellt (Jäger, S., 1995).

3 Das Duisburger Institutes für Sprach- und Sozialforschung (DISS) ist ein gemeinnütziger Verein, der seit 1987 Forschungs- und Handlungsprojekte zum Thema Einwanderung, Rassismus und Rechtsextremismus durchführt. In dieser Zeit entstand eine Vielzahl von Me- 
keitstheorie von Alexej N. Leontjew ein wichtiger Stellenwert zu (vgl. Jäger 2001; Leontjew, 1982).

Diskurse zu erfassen und zu analysieren, hatte Foucault sich vor allem auf der Diskursebene der Wissenschaften, insbesondere der Humanwissenschaften als Ziel gesetzt. Jäger stellt heraus, dass Diskursanalyse auch die Ebenen der Medien, der Politik, der Erziehung und des Alltags betrachten soll.

Der gesellschaftliche Gesamtdiskurs setzt sich zu jeder Zeit aus vielen Themen zusammen, die meist eng miteinander verwoben, sich aber analytisch recht deutlich voneinander abgrenzen lassen. Diese thematisch abgrenzbaren Teile des Diskurses kann man als Diskursstränge bezeichnen, z.B. der Diskursstrang Einwanderung, Flucht und Asyl; der Diskursstrang Frauen; der Diskursstrang Gesundheit, der Diskursstrang Krieg etc. Ein einzelnes angesprochenes Thema aus einem Diskursstrang bildet ein Diskursfragment. Die verschiedenen Diskursstränge sind eng miteinander verflochten und bilden so ein „diskursives Gewimmel“, dass man sich wie eine Art Wurzelwerk vorstellen kann, welches Diskursanalyse entwirren soll; hierbei ist darauf zu achten, wie sich die verschiedenen Diskursstränge beeinflussen, welche Überlappungen, Diskursverschränkungen sich ergeben.

Nach Link werden die Diskurse durch ein synchrones System kollektiver Symbolik (Sysykoll) zusammengehalten. Dies ist in allen Gesellschaften, wenn auch in unterschiedlichen Ausprägungen, zu beobachten. Sehr vereinfacht lässt sich das Grundschema der politischen Kollektivsymbolik der Bundesrepublik als Kreis skizzieren, der durch eine Horizontale und eine Vertikale, die sich in der Mitte schneiden, unterteilt ist. Dabei symbolisiert die Kreislinie die Grenze unseres Systems; die Vertikale ermöglicht die Abbildung eines linken Flügels, eines rechten Flügels und einer Mitte (also einer rudimentären politischen Taxonomie). Die Vertikale stellt einen oberen und unteren Abschnitt und wiederum die Mitte unseres Systems dar. In der Mitte sitzt das Herz, der Motor, das Energiezentrum. Unterhalb der Horizontalen wird die Finsternis des Untergrundes verortet, die des Urwalds und des Dschungels. Um Schieflagen zu vermeiden, ist unbedingt Ausgewogenheit geboten. Vor allem müssen die Aussenwände des Systems, in dem wir sitzen gegen das Chaos, etwa Flut, Wüste, Dschungel, Weltraum, Gewitter, Blitze, Brände, Stürme, Dunkelheit, Ungeheuer und Viren etc. abgedichtet werden. In der Mitte dieses Panzers sitzt das Subjekt: als ,Ich' und als ,Wir ${ }^{6}$ gleichermaßen einzig in seinem Eigentum - während von draußen die Bedrohung kommt (vgl. Link, 1984: 12f.).

Die in dieser Topik auftretenden und verwendeten Symbole werden als Kollektivsymbole bezeichnet, weil sie allen Menschen eines kulturellen Zusam- 
menhanges unmittelbar einleuchten. Durch Bildbrüche (Katachresen) werden die Symbole, die den unterschiedlichsten Bildspendebereichen entstammen, miteinander gekoppelt. So entsteht ein mäandrierendes Band, das sich durch die unterschiedlichen Diskursstränge zieht und dem gesellschaftlichen Gesamtdiskurs Stabilität verleiht, auch wenn dabei äußerst widersprüchliche Aussagen miteinander verbunden werden. Deshalb bezeichnet Link das System kollektiver Symbolik auch als „Kitt der Gesellschaft“ - dank dieses symbolischen Sinnbildungsgitters können wir uns in unserer Kultur stets zu Hause fühlen, obwohl wir in der realen Gesellschaft die Komplexität der politischen Zusammenhänge, Lebenssituationen, Arbeitsbedingungen etc. nur beschränkt durchblicken. Bei der Analyse der BILD-Berichterstattung ist die Analyse der Kollektivsymbolik im Hinblick die Konstruktion einer „Innenwelt“ und einer „Außenwelt“, eines „Wir“ und „die Anderen“, eines Feindes und eines Verbündeten von besonderer Bedeutung.

Diskurse spiegeln nicht die Wirklichkeit einfach wider, sie werden erst durch das tätige Subjekt wirklich, wobei sie aber auch die Gestaltung von Wirklichkeit, vermittelt über die tätigen Menschen bestimmen. Daher ist hier die Tätigkeitstheorie von A. N. Leontjew wichtig. Nach Leontjew ist das Verhältnis des Subjekts zur gegenständlichen Welt durch Tätigkeit vermittelt. Der Mensch lernt nicht durch reines Anschauen der Wirklichkeit, sondern indem er gestaltend und somit verändernd auf die Wirklichkeit einwirkt. Tätigkeit ist jedoch immer als Tätigkeit im Rahmen gesellschaftlicher Tätigkeit zu sehen eingebunden in den historischen Diskurs nach dessen Maßgabe Gesellschaften ihre Praxis organisieren; die gegebene soziale Wirklichkeit ist durch die Auseinandersetzung der Menschen mit dem „Rohstoff“ der Wirklichkeit bzw. mit den Produkten früherer menschlicher Tätigkeit entstanden. Wenn man nun das Verhältnis der Menschen zur natürlichen oder bereits sozial gestalteten Wirklichkeit in dieser Weise über Tätigkeit vermittelt begreift, wird deutlich, dass die Diskurse als Grundlage gesellschaftlichen Handelns ebenso Macht ausüben, wie Macht durch das individuelle Wissen jedes einzelnen Subjektes ausgeübt wird, das tätig auf die Wirklichkeit einwirkt (vgl. Leontjew, 1982).

Somit prägen und formieren Diskurse Realität nicht unmittelbar, sondern immer nur vermittelt über die dazwischentretenden tätigen Subjekte in ihren gesellschaftlichen Zusammenhängen als Produzenten der Diskurse und als Gestalter von Wirklichkeit. Das Individuum ist im Diskurs tätig, es ist auf den sozialen Diskurs verwiesen, wenn es tätig sein will bzw. muss, es ist in den sozialen Diskurs verstrickt, und es kann erst im Diskurs tätig sein, wenn es in diesen Diskurs eingebunden ist, ihm unterworfen ist, durch ihn subjektiviert wurde.

Diskurs wird hier nicht gleichgesetzt mit Gesellschaft - Diskurs wird als ein Bestandteil der Gesellschaft und als die bestimmende Kraft der Entwicklung 
gesellschaftlicher Wirklichkeit markiert; er resultiert aus den Kämpfen und Auseinandersetzungen tätiger Menschen in Gegenwart und Vergangenheit. Kein Individuum und keine Gruppe haben ihn genau so gewollt, wie er sich aktuell darstellt. Die Macht über die Diskurse ist in einer Gesellschaft ungleich verteilt, insofern geht auch die Macht der Diskurse mit individuellen Interessen nur mehr oder minder konform, anders gesagt: durch Macht über die Diskurse wird Herrschaft ausgeübt. Zusammenfassend kann man sagen: Das einzelne Subjekt konstituiert den Diskurs nicht, vielmehr wird das Subjekt vom Diskurs konstituiert, Diskurs ist überindividuell.

Das Subjekt nimmt im Gewimmel der Diskurse eine bestimmte Position ein - die Subjekt- oder Diskursposition. Somit wird das Subjekt keinesfalls geleugnet - es wird lediglich das Vorhandensein eines autonomen Subjektes außerhalb jeglicher Diskurse bestritten. In seinen späteren Arbeiten bezeichnet Foucault das Subjekt sogar als das allgemeine Thema seiner Forschung. Er fragt, auf welche Weise das Subjekt zum Unterworfenen - eben zum Subjekt - geworden ist und wie es sich von dieser Unterwerfung, von der Herrschaft, die über es ausgeübt wird, befreien kann (Foucault, 1987: 243ff.).

Die Medien lassen sich vor diesem Hintergrund als eine Art „Brennglas“ fassen, das vorhandenes Wissen spezifisch ,bündelt" und dieses Wissen an ein Massenpublikum weitergibt. Sie geben Argumentationslinien vor und produzieren Applikationsvorgaben für individuelle und kollektive Subjektbildung (vgl. Jäger, M. et al., 1997).

Unter Anwendung des diskursanalytischen Instrumentariums sollen die Argumentationsstrategien, die Symbolik und die Implikate der BILDBerichterstattung in zwei Phasen betrachtet werden:

Vom 12.9.2001 bis zum 06.10.2001 (am 7.10.2001 begann der militärische Einsatz der US-Truppen mit Unterstützung Großbritanniens gegen Afghanis$\tan )$.

Vom 8.10.2001 bis zum 7.11.2001 (am 7.11.2001 wurde die Entscheidung des deutschen Bundeskanzlers bekanntgegeben, 3900 Soldaten zur Unterstützung des militärischen Einsatzes zur Verfügung zu stellen)

\section{Diskursanalytische Überlegungen zur BILD-Berichterstattung vom 12.9.01 - 6.10.01}

Am 12.9.01 berichtet BILD auf sieben Sonderseiten, ausgiebig bebildert. Vom 13.9. bis einschließlich 18.9.01 erschienen die Ausgaben unter Einbeziehen der amerikanischen Flagge in das BILD-eigene Logo ${ }^{4}$, die symbolische Antwort auf die Solidaritätsbekundung des früheren amerikanischen Präsidenten

4 Ähnlich auch die Diskursstrategie der britischen Tageszeitung „The Sun“. Hier wurden zu Beginn der Angriffe der USA auf Afghanistan unter Beteiligung Großbritanniens der Union Jack und Stars 'n' Stripes nebeneinander projiziert in das Logo der „Sun“ einbezogen. 
John F. Kennedy. Sein Bekenntnis: „Ich bin ein Berliner“ kann heute - so legt der Mediendiskurs der BILD nahe - mit dem umfassenderen Bekenntnis: „Wir sind alle Amerikaner" beantwortet werden. Die Implikate dieser Diskursstrategie sollen im folgenden dargestellt werden. Vier Themenbereiche traten bei der Analyse in den Vordergrund.

\section{Terror versus Krieg}

Der Terrorismusexperte Bruce Hoffman grenzt Terror von Krieg ab, indem er darauf verweist, dass beide Arten von Gewalt sich dadurch unterscheiden, dass der Krieg Regeln kennt. „So legen völkerrechtliche Konventionen das Verbot fest, Zivilisten als Geiseln zu nehmen und gegen sie Racheakte zu richten. Entsprechende Vergehen können als Kriegsverbrechen geahndet werden. Zum internationalen Terrorismus, so merkt Hoffman an, gehöre es allerdings, „sich an solche Regeln der Kriegsführung und den entsprechenden Verhaltenskodex“ nicht gebunden zu fühlen. Hoffman definiert „den Terroristen“ als „,im Kern gewalttätigen Intellektuellen, ... der dazu bereit ist, Gewalt zur Erreichung seiner Ziele einzusetzen, Aufmerksamkeit zu schaffen und vor allem - Angst zu erzeugen." (Arning, FR 27.9.01, S. 2).

Am 12.9.2001, dem Tag nach dem Anschlag, berichtet BILD auf sieben Sonderseiten mit ausgiebiger, zum Teil farbiger Bebilderung. Die Titelseite zeigt einen Turm des World-Trade-Centers mit seitlicher Explosionswolke, Rauch und Feuer in den darüberliegenden Stockwerken. Weiter unten wird das Einfliegen des Flugzeuges bis hin zur Explosion im zweiten Turm in einer Dreier-Sequenz abgebildet. Ein Schwarz-weiß-Foto zeigt Überlebende voller Staub - in Schutt und Asche.

Die auf rotem Grund gedruckte Überschrift zum Titel - „Tausende Tote in Amerika! Die Welt in Angst! Gibt es Krieg?"“ - umreißt die wichtigsten Bedeutungsfelder - Tod, Angst, Krieg. Die Schlagzeile selbst ist ein Stoßgebet: „Großer Gott steh uns bei!“

Im Hinblick auf das Thema „Krieg“ wird hier erst mal eine Frage gestellt, die aber auf der gleichen Seite im Kommentar beantwortet wird: „Kriegserklärung an die Menschheit“. Amerika wird hier als Pars pro toto verwendet, es steht für die ganze Welt. Logisch weiter nachvollzogen ist somit der Angriff auf die Menschen in Amerika ein Angriff auf die Menschheit und damit eine Kriegserklärung an die gesamte Menschheit.

Im einleitenden Satz wird das Geschehene als „der schlimmste Terroranschlag in der Geschichte der Menschheit" beschrieben. ,... um 8.48 Uhr attackierten Terroristen das Herz der Welt.“ Im weiteren sollen „Kriegsschiffe und Bodentruppen jetzt die USA mit Raketenabwehrsystemen schützen.“ Der muslimische Extremist Osama bin Laden, der zurückgezogen in den Bergen Afghanistans lebt, wo er das Gastrecht der Taliban genieße, wird als Drahtzieher 
der Anschläge genannt. Gleichfalls wird die Reaktion von Bundeskanzler Gerhard Schröder bekannt gegeben: „Er bezeichnete die Terroranschläge als ,Kriegserklärung gegen die gesamte zivilisierte Welt'.“

Es wird also einerseits die Perspektive erweitert - von Amerika auf die gesamte Welt - und dann wieder verengt auf Deutschland, von wo aus wieder eine teilweise Erweiterung auf die „gesamte zivilisierte Welt“ stattfindet. So wird in der perspektivischen Führung der Argumentation die Erweiterung und Verengung nachvollzogen, die de facto an einem funktionierenden Herzen besteht. Im Rhythmus des Herzschlages wird eingeschworen auf eine Erkenntnis, die für das weitere Vorgehen der USA von grundlegender Bedeutung ist: Hier handelt es sich nicht um „bloßen“ Terror, hier handelt es sich um Krieg. Dabei ist die Herzsymbolik von besonderer Wichtigkeit.

Wenn mit dem Anschlag auf das World Trade Center und das Pentagon das „Herz der freien Welt“, wie BILD schreibt, getroffen ist, handelt es sich um einen absoluten Notstand, auf den reagiert werden muss. Die „freie Welt“, durch Schröder weiter spezifiziert als „die zivilisierte Welt“, steht vor dem Zusammenbruch, dem Ausbluten, dem Tod. Damit ist höchster Handlungsbedarf gegeben.

Auf den folgenden Seiten wird die Herzsymbolik, z.T. in Zusammenhang mit „Blut, Blutkonserven“ immer wieder aufgegriffen. So unter dem doppelseitigen Titel „Um 8.48 Uhr explodierte der 1. Jet im World Trade Center“. Unter der Rubrik „Das Protokoll“ wird der chronologische Ablauf des Geschehens von $8.40 \mathrm{Uhr}$ bis 13.20 Uhr wiedergegeben. Er endet mit den Worten „Blutkonserven gehen aus, Kliniken bitten die Bevölkerung in New York und Washington um Spenden“. Die Chronologie genau hier enden zu lassen, verweist auf den symbolischen Gehalt der Aussage - das Blut geht aus, der Tod naht, nur Spenden, also fremde Unterstützung, d.h. übertragen auf den Gesamtzusammenhang auch deutsche Unterstützung - kann die Situation / das Leben vielleicht noch retten.

Auf S. 3 beginnt dann die Suche nach rationalen Erklärungen „Warum die Türme so schnell einstürzten". Neben der Chronologie des Einsturzes findet sich hier die Erklärung des statischen „Schneeballeffektes“, der laut Aussage der Statiker stattfinden musste. Damit wird jedes Argument entkräftet, das lauten könnte, die Attentäter hätten nicht damit gerechnet, dass die Gebäude in so kurzer Zeit völlig in sich zusammenstürzen und so viele Menschen unter sich begraben. Das World-Trade-Center, der Titel lautet „World Trade Center - Wahrzeichen New Yorks“, „war das Symbol der Kraft und Stärke der USA. Das wirtschaftliche Herz Amerikas.“ Hier klingt ein neuer Aspekt an - die wirtschaftlichen Auswirkungen des Anschlags. Auch hier wird die Herzsymbolik in den Vordergrund gestellt. Hieran schließt drei Seiten weiter der Bericht „Nach der ersten Explosion stürzten die Börsenkurse“ ein. Zwar wird hier nicht mehr die Herzsymbolik eingesetzt, doch „der Terroranschlag 
... riss gestern weltweit die Börsen in die Tiefe. ... Nachricht ... wie eine Schockwelle. ... ,panikartige Verkäufe auf breiter Front' “ Hier wird, kollektivsymbolisch aufgeladen, der Absturz des Systems beschrieben, der Erdrutsch in ungeahnte Tiefen, in das bedrohliche Chaos, die zerstörende Erschütterung des geschlossenen Systems, das über die äußere Grenze hinaus, über die Front, Besitz abstößt - wohin? Auch hier wird - wenn auch nicht mit den entsprechenden Termini - ein Ausbluten beschrieben, der Verlust von Reichtum, von Geld. Gleichzeitig erfolgte eine Aufnahme der Kriegssymbolik „auf breiter Front“ - und somit schließt sich der symbolische Aussagekreis: „im Herzen getroffen, vor dem Ausbluten“ hin zu „hier handelt es sich um Krieg“.

Die zitierten Textstellen stehen nur als Beispiele für die in der Berichterstattung noch häufig aufgegriffene Herzsymbolik. Sie bilden das Zentrum der mediopolitischen Strategie der BILD-Zeitung - das Einschwören ihrer Leser auf eine notwendigerweise kriegerische Antwort auf ein als Krieg definiertes Geschehen.

\section{Der Pearl-Harbor-Vergleich}

Der Angriff der Japaner auf die amerikanische Flotte von Pearl Harbor, der nach offizieller Lesart überraschend und ohne Vorankündigung stattfand, was inzwischen unter Historikern kontrovers diskutiert wird - und über 2500 Opfer forderte, brachte die Amerikaner 1941 in den 2. Weltkrieg. Mit dem Vergleich des jetzigen Geschehens mit Pearl-Harbor wird der Definition der Terroranschläge als Krieg zugearbeitet. Bereits im Kommentar „Kriegserklärung an die Menschheit“ auf der Titelseite heißt es: „Ein neues Pearl Harbor. Doch diesmal ist nicht die Flotte der USA getroffen worden, sondern Amerika, seine Bürger, seine Häuser.“ (BILD, 12.9.01, S. 1). Die Häuser, die eigentlich Schutz gewähren, das Innen, die Wände, die vor dem Chaos abschotten, sind zerstört und mit ihnen die Menschen. Der kriegsgleiche Charakter des Geschehens wird ausgebaut und damit der Weg für einen kriegerischen Gegenschlag geebnet.

Peter Scholl-Latour geht unter dem Titel „Gibt es jetzt Krieg, Herr SchollLatour?“ darüber hinaus. „Mit den Kamikaze-Angriffen auf das Herz von New York wollten sie das Symbol einer Globalisierung treffen, die in Wirklichkeit eine globale Amerikanisierung ist. ... Dagegen war der vernichtende Angriff der japanischen Flotte von Pearl Harbor im Dezember 1941 eine Randerscheinung." Die im Titel gestellte Frage wird in dem Text nicht explizit beantwortet; das ist auch nicht nötig, denn implizit besagt die Bezeichnung von Pearl Harbor als „Randerscheinung“, dass es sich bei den jetzigen Geschehnissen um Entscheidenderes handelt, und wenn Pearl Harbor die Amerikaner schon in den 2. Weltkrieg gebracht hat, dann muss die Frage nach Krieg jetzt nicht mehr gestellt werden: Es herrscht bereits Krieg. 
Die Bezeichnung der Globalisierung als „globale Amerikanisierung" bringt einen weiteren Aspekt ins Spiel. Es wird hier ein Sagbarkeitsfeld eröffnet, welches Globalisierungsgegner mit Anti-Amerikanern gleichgestellt und somit Globalisierungsgegner mit Terroristen identifiziert; ein Sagbarkeitsfeld, welches gleich auch bearbeitet wurde in der Aussage des italienischen Ministerpräsidenten Silvio Berlusconi, der „eine merkwürdige Übereinstimmung zwischen den Globalisierungsgegnern und den islamischen Terroristen sieht. Beide seien Gegner der westlichen Zivilisation." (FR, 27.9.01, S. 1). Weitergedacht eröffnet dies gegen Globalisierungsgegner, wie etwa gegen die Menschen, die bei dem G-8 Gipfel in Genua protestietren und arrestiert, noch ganz andere Möglichkeiten des Vorgehens...

\section{Der Hitler-Vergleich}

In der Kolumne „Post von Wagner“ findet ein Hitler-Vergleich statt: „Machen wir uns nichts vor, es ist der dritte Weltkrieg. Es ist wie bei Hitler. Die Weltmacht Terror überfiel uns im Schlaf. Tausende Tote, Sekretärinnen, Angestellte, Kinder, Touristen. Die Symbole Amerikas in Schutt und Asche.“ Was „wie bei Hitler“ ist, wird nicht klar. Dass die „Weltmacht Terror“ uns „bei Hitler“ im Schlaf überfallen hat, kann wohl kaum gemeint sein - das wäre eine völlig ahistorische Perspektive. Auch ist die Anzahl der Toten des Hitler-Regimes um ein Vielfaches höher als die des Anschlages. Sachlich ist somit dieser Vergleich nicht zu rechtfertigen. Inhaltlich wird aber nahegelegt, dass es sich bei dem Anschlag um einen geplanten, kontrollierten Massenmord handelte, der mit den rassistisch motivierten Massenmorden des dritten Reiches vergleichbar ist und somit nur eine Deutung der Ereignisse und eine Antwort zulässt: Krieg.

\section{Das eskalierende Kriegsszenario}

Die BILD vom 13.9.01 titelt mit „Der Tag danach“ auf einem ganzseitigen Foto der noch rauchenden Trümmer. „Nato erklärt Verteidigungs-Fall. Auch Deutschland dabei“. In Anspielung auf die filmische Auseinandersetzung mit dem Atomkrieg „The Day After“ wird hier weiter der Krieg beschworen und Deutschlands Rolle im Nato-Verteidigungsfall beschrieben. Dieser NatoVerteidigungsfall wird hier bereits als erklärt beschrieben - in vorauseilendem Gehorsam gegenüber den Ereignissen. Tatsächlich wurde erst am 2.10.01 erklärt, dass der Nato-Verteidigungsfall vorliege. Der Umgang mit der Thematik in der BILD-Zeitung zeigt in aller Deutlichkeit mit welcher Dringlichkeit „Krieg“ zeitlich nah zu den Terroranschlägen diskursiviert wurde. Unter leichter Rücknahme dieser Dringlichkeit - die Vermutung liegt nahe, dass dies eine Folge des noch nicht stattgefundenen militärischen Schlages der USA ist - wird am 18.9.01 die Frage gestellt: „Ist der Krieg noch zu verhindern?“ Am 19.9.01 befragt BILD dann den deutschen Kanzler: „Kanzler, wie 
schlimm ist es für uns?"“, der in der darauffolgenden Ausgabe die Deutschen beruhigt: „Wir haben keinen Krieg“. Das übereilt entworfene Kriegsszenario wird sodann am 21.9.01 durch ein stärker rechtsstaatliches Szenario begrenzt, bei dem nun „Beweise“ gegen die Terroristen im Vordergrund stehen. Am 25.9.01 verschwinden die Terroranschläge dann aus dem Haupttitel der BILD-Zeitung, um am 29.9.01 mit einem weiteren Beweis: „Der Terror-Befehl - Bekennerschreiben von Todespilot Atta entdeckt“" zurückzukommen und das Kriegsszenario - nun unter rechtsstaatlichen Aspekten - wiederzubeleben: „Der Countdown läuft - Nur noch 24 Stunden bis zum Schlag gegen Afghanistan" (1.10.01) und Unterstreichung der Dringlichkeit bzw. der Unabwendbarkeit eines militärischen Gegenschlages in der Schlagzeile: „Angst vor neuen Anschlägen nach US-Angriff“" am 2.10.2001. Diese Meldung ist doppeldeutig. Sie kann einmal so gelesen werden, als habe bereits ein US-Angriff stattgefunden und nun habe man Angst vor neuen Anschlägen, sie kann aber auch so gedeutet werden, dass die Angst vor neuen Anschlägen im Falle eines US-Angriffs besteht, wobei die erste Lesart die naheliegendere ist. Da bis zu diesem Datum noch kein US-Angriff stattgefunden hatte, zeigt sich hier wieder die voreilige Beschreibung eines Ereignisses, welches noch gar nicht stattgefunden hat. Durch diese Strategie wird die Unausweichlichkeit im Hinblick auf den Krieg in den Vordergrund gestellt.

\section{2. "Wir und die Anderen”}

Der Titel der BILD-Zeitung vom 12.9.01 verweist auf eine weitere Ebene, die an der Schnittstelle der Diskussion um „den Krieg“ liegt. Der Titel verweist auf die stattfindende Polarisierung der Welt auf der Grundlage einfacher binärer Oppositionen: „Großer Gott, steh uns bei!“ Auf den ersten Blick und unter Einbeziehung der Überschrift „Die Welt in Angst“, könnte es sich hier um ein allgemein formuliertes, nicht polarisierendes Stoßgebet angesichts des erfahrenen Horrors und der nachfolgenden Angst handeln - „, steh uns bei“, uns, der Welt. Doch in dem Text darunter geht es weiter: „Wir haben alle Angst ... attackierten Terroristen das Herz der freien Welt. ... Als Drahtzieher gilt der saudische Terror-Chef Osama bin Laden. Entsetzen, Trauer, Wut vereinen die restliche Welt. ... Bundeskanzler Schröder ... bezeichnete die Terroranschläge als ,Kriegserklärung gegen die gesamte zivilisierte Welt.““ wird „die Welt“ von ihrer Gesamtheit reduziert auf die „freie, restliche, zivilisierte" Welt. Damit wird gleichzeitig eine Gegenwelt aufgebaut, die es zu bekämpfen gilt: die „unfreie, unzivilisierte“ Welt - eben „der Westen“ und „der Rest“, „Wir“ und „die Anderen“ und darüber hinaus „die Zivilisierten“ und „die Barbaren“, die Christen und die Muslime. Vor diesem Hintergrund erscheint der Titel in einem anderen Licht. „Wir Christen rufen unseren Gott um Beistand an.“ Und mit dem Anruf „steh uns bei“ sind die Amerikaner 
gemeint, von denen es heute viel mehr gibt, als gestern noch, denn, so heißt es auf Seite 2: „Laßt uns diesen Satz in unser Universum hinausschreien. Wir sind alle Amerikaner." Und in den folgenden Tagen wird auf diesen Satz eingeschworen. Am 13.9., 14.9. und 15.9. findet sich auf insgesamt 15 Innenseiten, die über die Anschläge berichten, eine Spalte mit dem Titel: „Jetzt sind wir alle Amerikaner, weil ..." Neben den Bildern von Prominenten und Nicht-Prominenten sind dann die Begründungen angegeben.

\section{Der Feind und das Feindbild}

Die Frage nach den Schuldigen beantwortet die BILD-Zeitung vom 12.9.01 schnell: „Wer ist der Terror-Chef“ und „Wer sind die Selbstmord-Piloten?" Auf S. 4 zeigt eine Abbildung Osama bin Laden sitzend mit weißer Kopfbedeckung, langem Bart und einer Waffe auf den Schenkeln, auf der seine beiden Hände ruhen. „Die USA vermutet die Terror-Organisation des MoslemExtremisten Osama bin Laden hinter den Anschlägen." Zitiert werden Mitteilungen über geplante Anschläge und die Fatwa, einen religiösen Erlass, vom Februar 1998: „Wir rufen mit Gottes Hilfe jeden Moslem, der an Gott glaubt und der belohnt werden will, weil er an Gottes Gebote glaubt, auf, die Amerikaner zu töten und ihr Geld zu rauben, wo immer es ist." Das kann nicht der Gott sein, den „wir“ auf der Titelseite angerufen haben. Der Feind ist benannt, das Feindbild personalisiert, die Person verbreitet Angst, und das Feindbild ist übertragbar. Osama bin Laden wird in eine binäre Opposition zum amerikanischen Präsidenten George W. Bush gestellt. Auf der BILDAusgabe vom 18.09.01 erscheint bin Laden mit gelassenem Gesichtsausdruck, in Großaufnahme einem kleinen Bild Bushs gegenüber, der sich mit geballter Faust „kämpferisch“ zeigt. Die Angst vor dem Feind wird auf seine Religion übertragen - der Islam, die Muslime geraten so in die Ziellinie der notwendigen Bekämpfung, ist es doch ihr Gott, der dazu beitragen soll, die Amerikaner, also „uns“ zu töten. Bei der Beschreibung der Selbstmord-Piloten wird hervorgehoben, dass „verbrämt mit religiösen Motiven ... die Waffe des Selbstmordterrorismus eine lange Tradition in der islamischen Welt hat. (Die Attentäter) ... verstehen sich als islamische ,heilige Krieger ${ }^{6}$... als Märtyrer verehrt. ... Im islamischen Paradies ist ihnen ein Ehrenplatz innerhalb der Heiligenhierarchie sicher.“

In diesem Zusammenhang - Extremismus und Feindbild - steht auch die Berichterstattung „Palästinenser-Extremisten tanzten auf den Straßen“. Eine Schwarz-Weiß-Aufnahme zeigt jubelnde und tanzende Kinder „Palästinensische Jugendliche tanzen auf der Straße, nachdem sie von dem Anschlag erfahren haben." Links ist eine Abbildung von Israels Regierungschef Sharon, der „Israels Reserve-Piloten in die Kasernen“ rief. Rechts im Bild findet sich der Palästinenser-Präsident Jassir Arafat: „Verurteilt offiziell den Anschlag.“ 
Die Al-Aksa-Intifada stand kurz vor ihrer ersten Jährung; die geplanten Friedensgespräche zwischen Arafat und dem israelischen Außenminister Shimon Peres wurden verschoben. Die Polarisierung wird deutlich: von Amerika unterstützte Israelis versus extremistische Pälästinenser, deren Präsident den Anschlag - zwar oder nur? - „offiziell verurteilt“. In dem Artikel wird beschrieben, dass palästinensische Sicherheitskräfte vergeblich versuchten, die Feiernden (hauptsächlich Kinder) von der Straße zu jagen, wo Süßigkeiten verteilt wurden und „Gott ist groß“ skandiert wurde. Des weiteren wird beschrieben, dass ein palästinensischer Guerilla-Führer sich mit den unbekannten Terroristen solidarisiert, weil die Bush-Regierung die Israelis unterstützt.

Inbesondere durch die doppeldeutige Beschreibung der Distanzierung Arafats in dem Wort „offiziell“, welches die Frage nach der „inoffiziellen“ Version zulässt, werden hier ,die Palästinenser ' mit ,extremistischen Palästinensern homogenisiert und somit alle Palästinenser auf die Seite der terrorbereiten „Anti-Amerikaner“ gestellt. Die Mitteilung „Die demokratische Front für die Befreiung Palästinas (DFLP) und die radikal-islamistische Palästinenserorganisation „Hamas“ bestritten Meldungen über eine Tatbeteiligung“ stellt Meldung und Widerspruch in einen Dialog, der freie Interpretationen zuläßt und bringt den möglichen Netzwerkcharakter bzw. lose Allianzen des islamistischen Extremismus in den Vordergrund.

Der Aspekt des globalisierten Netzwerkcharakters der Terroranschläge weicht das personalisierte Feindbild ,Osama bin Laden' teilweise auf. Dies deutet sich bereits in der Berichterstattung vom 24.9.01 über sein Verschwinden und seine mögliche Veränderung an. „Sogar den Bart abrasiert? Bin Laden spurlos verschwunden." Eine Computersimulation auf der 2. Seite zeigt Bin Laden ohne Bart und beschreibt, dass 11.000 Terroristen von bin Laden ausgebildet wurden. Die Grenzen zwischen personalisiertem Feindbild und ,subjektlosen Feindmassen" verschwimmen, wie auch der Kommentar auf der Titelseite andeutet: „Kriegserklärung aus dem Dunkel“. Hier kommt, kollektivsymbolisch gesehen, die Gegenwelt zu der geschlossenen Innenwelt zum Tragen: das Chaos, das Dunkel, die Bedrohung, die sich auch in ihrer metaphysischen Kraft auf die Innenwelt auswirkt und „die Menschen ... wie Gespenster, von Schmutz bedeckt, weinend und taumelnd“ („Um 8.48 Uhr ...“, BILD, 12.9.01, S. 2) zurücklässt.

Es werden in großem Umfang Personalisierungen eingesetzt, Einzelschicksale dargestellt - auch hier wieder prominenter und nicht-prominenter Personen, um den LeserInnen möglichst umfangreiche Identifikationsmöglichkeiten zu bieten. So lautet der Titel vom 15.9.01 „Angst“ und zeigt die Großaufnahme einer weinenden jungen Frau, die mit einem Bild in der Hand ihren Verlobten sucht. Der Bericht wird auf S. 5 - „Sie suchen ihre Liebsten“ - fortgesetzt. Das Bedrohungsszenario wird nun weitgehend auch auf Deutschland übertragen: „Attentäter lebte 8 Jahre in Deutschland - Terror-Bestie“ (BILD, 
14.9.01, S. 1). „Das Massaker von New York wurde in Hamburg geplant“ (ebd., S. 2). Die diffuse Bedrohung, der „Feind aus dem Dunkel“ wird beschrieben: „Das geheime Leben der Terroristen in Hamburg.“ (BILD, 15.9.01, S. 4). Am 17.9.01 wird dann die Verbindung zwischen Deutschlands Opfern und Deutschlands Verantwortung geschaffen: „270 Deutsche unter den Trümmern.“ „Terror-Pilot lebte von deutschem Stipendium“. Darüber steht: „Auch deshalb müssen wir an der Seite der Amerikaner stehen." (BILD, 17.9.01, S. 1)

\section{Die Apokalypse}

Das Metaphysische, der Grenzbegriff menschlicher Erfahrungsmöglichkeiten, dem auch die Welt des Geistes und des Glaubens zugeordnet werden kann, da sie nur gedacht und nicht wirklich erfasst werden kann - das Übersinnliche scheint in Zusammenhang mit den Terroranschlägen von besonderer Bedeutung zu sein. Die immer stärker werdende Polarisierung spitzt sich auf zwei große Glaubensrichtungen der Welt zu - das Christentum und den Islam - und birgt die Gefahr, die extremistischen, fundamentalistischen Glaubensgruppen mit dem gesamten Glauben gleichzusetzen. „Die Kriegserklärung aus dem Dunkel“ (BILD, 12.9.01, S. 1), die zum „Inferno“ führte, nimmt apokalyptische Ausmaße an: „... möchte in meiner Panik Zuflucht zu Gott suchen. Der Rauch ist groß, ich sehe ihn nicht mehr. Die Reiter der Apokalyse sind zu euch und unter uns gekommen. Der Teufel lacht, während wir weinen." (BILD, 12.9.01, S. 2). Beschrieben wird hier ein Weltuntergangsszenario, gegen das es keine Hilfe gibt, in dem „das Böse“ gewinnt.

Apokalyptische Vorstellungen gibt es unter anderem sowohl im christlichen Glauben als auch im Islam. Eine weiterführende Auseinandersetzung damit würde den Rahmen dieser Analyse überschreiten. Es soll allerdings hinsichtlich der zitierten Textstellen auf den Bibeltext „Offenbarung an Johannes“ hingewiesen werden, der die Apokalyse und die vier Apokalyptischen Reiter, die Pest, Krieg, Hungersnot und Tod symbolisieren, beschreibt, sowie auf das Buch Daniel des Alten Testaments und das Matthäus-Evangelium (Kap. 24-25). ${ }^{5}$

Wenn Peter Scholl-Latour seinen Text am 12.9.01 auf S. 5 mit den Worten beginnt: „Als ,Koloss auf tönernen Füßen', so ist die Weltmacht USA plötzlich dem weltweiten Fernsehpublikum erschienen“, so rekurriert er auf den Traum des Königs Nebudkadnezzar im Buch Daniel. „... von dieser apokalyptisch anmutenden Horror-Tat“, so spezifiziert Scholl-Latour die Zusammenhänge weiter, bis hin $\mathrm{zu}$ „Was so erschreckend ist an diesen geballten Vernichtungsschlägen des „Fünften Reiters“ ist die Kombination von religiösem Fanatismus, der in Selbstaufopferung gipfelt, mit einer logistischen Infra-

5 Der amerikanische Religionswissenschaftler David Cook verweist auf die Notwendigkeit, sich, will man den modernen Islamismus verstehen, mit seinen apokalyptischen Wurzeln zu beschäftigen. (Die Zeit, 20.9.01, S. 15). 
struktur, die man Orientalen normalerweise nicht zutraut." Nach dieser bemerkenswerten Äußerung statuiert er weiter: „Es kann sich nur um eine Allianz todesbesessener Gotteskrieger einerseits und hochvermögender Finanziers andererseits handeln, die in den USA über eine ausreichende Infrastruktur und hochgeschultes Personal verfügen." Über die eigentlichen apokalyptischen Vorstellungen hinaus wird hier der „5. Reiter“ beschrieben - ein Netzwerk von besessenen Gläubigen und ,dem Kapital'.

Die diffuse und undurchschaubare Bedrohungssituation wird zugespitzt: FBI und CIA haben versagt: „Die Kamikaze-Attentäter trafen nicht nur das Herz Amerikas, sondern zerstörten auch den Mythos CIA und FBI“, während Osama bin Laden den Attentätern gratuliert (BILD, 13.9.01, S. 5). Es verdichtet sich das symbolische Bild der Auflösung des schutzgebenden Innenraumes, des geschlossenen Systems durch das Eindringen der Feinde. Die Abwehrmechanismen sind zerstört. „Krieg gegen Taliban könnte Jahre dauern“ lautet eine Ankündigung am 17.09.01 unter der Beschreibung: „Die Woche der Apokalypse" auf S. 2. Das apokalyptische Szenario, welches sich in der BILDBerichterstattung durchgängig findet, wird noch weiter zugespitzt und der symbolische Kreis - Körper (Amerika, „zivilisierte Welt“ - Virenangriff (Bin Laden, Taliban, „das Böse“) - Tod (,ins Herz getroffen“), der sich in der BILD-Zeitung aufzeigen läßt, hat seine Entsprechung auf der Realitätsebene: „Ungleich verheerendere Waffen sind längst entwickelt, Waffen der atomaren, chemischen und bakteriologischen Kriegsführung. Und auch auf sie wird das Böse eines Tages Zugriff haben (soweit es nicht schon Zugriff hat).“ (BILD, 17.9.01, S. 2).

Am 4.10.01 setzt sich die Titel-Berichterstattung mit der Frage des TVVerbots für den TV-Moderator Ulrich Wickert auseinander, der in einem Interview für die Illustrierte Max Bush und bin Laden verglichen hatte: „Bush ist kein Mörder und Terrorist. Aber die Denkstrukturen sind die gleichen.“ Die aufgeführten Zitate und Wickerts Stellungnahme distanzieren sich stark von diesem Vergleich und führen zurück auf die eingeschworene Diskursstrategie: „Die Amerikaner müssen jetzt spüren, dass wir bei ihnen sind“ - so wird Helmut Kohl zitiert. Weiter auf der Titelseite: „Die USA kreisen Afghanistan ein“. Hier wird darauf hingewiesen, dass die USA „erstmals stichhaltige Beweise für die Täterschaft bin Ladens vorlegten.“ Diese werden auf Seite 3 als Telefongespräche und Zahlungen von Bin Ladens Terror-Organisation „El Kaida" in Zusammenhang mit den Attentaten beschrieben. Ein Foto zeigt, wie „fanatische Muslime in Pakistan“ mit Bin-Laden-Fotos demonstrieren und weiter unten wird ein „Palästinenser-Blutbad unter israelischen Siedlern“ aufgeführt. Die Argumentationsstrategie manifestiert sich in der Berichtanordnung und Aufmachung und scheint auf eine Homogenisierung von Extremisten und Palästinensern angelegt zu sein. Ein weiterer kleiner Artikel, der eine zweite Terrorwelle als geplant beschreibt - die Sprengung des Sears 
Towers in Chicago mit möglichen 20.000 Toten sowie ein Attentat auf die US-Botschaft in Paris durch einen ehemaligen Fußballspieler von Fortuna Düsseldorf - wirkt seltsam losgelöst - es gibt keinen Hinweis darauf, woher diese Informationen kommen und wie die Attentate verhindert werden konnten. Der Bericht beschwört das nicht enden wollende Terrorszenario weiter. Nun ist es schon zur Durchsetzung eines unserer wichtigsten deutschen nationalen Symbole - des Fussballs - mit Attentätern, also Viren, gekommen. Während die Rettung einer Katze aus den Trümmern und „New York wird wieder blühen, schöner denn je“ (BILD 5.10.01, S. 5) Positives für die amerikanische Zukunft verheißt, wird am 6.10.01 die Kritik des israelischen Premierministers Ariel Scharon aufgegriffen, der sein Land durch Bündnisbereitschaft der USA „mit Feinden Israels“ im Anti-Terror-Pakt verraten sieht. Ein Video, welches Bin Laden feiernd im Kreis seiner „Gotteskrieger“ zeigt, soll mehr Transparenz in das Feindbild bringen. Der "Chefstratege des Terrorfürsten " und sein bisheriger Beitrag zu terroristischen Handlungen werden gezeigt: „Der Schlächter und sein Denker. (...) Jetzt kennt die ganze Welt das Gesicht von Ayman el Zawahiri. Auch er wird jetzt von Amerika und seinen Verbündeten mit allen Mitteln gejagt." Mit dieser Diskursstrategie wird verdeutlicht, wo die Freunde und wo die Feinde Amerikas sind.

\section{Vorläufiges Fazit - Phase I}

Am Tag nach den Anschlägen wird durch die BILD-Zeitung der Grundstein für eine diffuse, hochgradig alarmierende Bedrohungssituation gelegt, die durch die neue Art von Terror und die Richtung, diesem zu begegnen, entstanden ist. Die Berichte der darauf folgenden Tage bauen darauf auf und legen Zeugnis ab über eine auf Eskalation angelegte Diskursstrategie - von Terror zu Krieg, der von allen Mitgliedern der „westlichen Welt“ und für die BILD-Leser vor allem von den Deutschen mitgetragen werden muss.

In vorauseilendem Gehorsam gegenüber den Ereignissen wird laut BILD bereits am 13.9. der Verteidigungsfall der Nato erklärt und statuiert: „Deutschland ist dabei." Schnell sollte auf den ganz offenbar erwarteten eiligen militärischen Schlag der USA gegen vermeintliche Attentäter oder Afghanistan als deren Gastgeberland eingestimmt und dieser legitimiert werden. Das es vorläufig nicht dazu kam, führte dann an den nachfolgenden Tagen zu einer in gewisser Weise verunsicherten Berichterstattung, die über apokalyptische Bilder hin zur Darstellung von Einzelschicksalen und dann ab 22.9.01 zum Rücklaufen der Thematik beitrug. Zu einer erneuten Zuspitzung des Kriegsszenarios in der Berichterstattung kam es am 29.9.01, als ein Bekennerschreiben des Selbstmordattentäters Atta veröffentlicht wird, womit sich jetzt unter Aspekten der Rechtsstaatlichkeit die Beweislage verbessert und nun „Der Countdown läuft.“ (BILD, 1.10.01, S. 1). 


\section{Diskursanalytische Überlegungen zur BILD-Berichterstattung vom 8.10.01 - 7.11.2001}

Am 7.10.01 beginnt der militärische Einsatz der USA mit der Unterstützung Großbritanniens. Eine Analyse der Berichterstattung ab 8.10.01 soll zur Beurteilung des Stellenwerts der bis zum Kriegsbeginn pulsierenden Berichterstattung - eskalierend, als ein früher militärischer Schlag der US-Streitkräfte erwartet wurde und, als dieser nicht eintrat, zurückgefahren auf ein rechtsstaatliches Szenario - im Fortgang der Ereignisse beitragen. Wie greift die BILDBerichterstattung auf, dass der Krieg keinen schnellen Erfolg bringt, dass der amerikanische Verteidigungsminister daran zweifelt, ob Bin Laden jemals gefasst werden kann, dass Militärexperten feststellen, die Taliban seien „zähe Kämpfer"?

Die Titelseite des 8.10.01 zeigt die grünen Blitze des Abwehrfeuers der Taliban unter der Überschrift „Der Angriff“ - „Der Feldzug gegen den Terror hat begonnen“. Die Rede von Präsident Bush wird zitiert. Unter dem Motto „Der Ernstfall ist da“ werden die Notwendigkeit des Krieges und seine möglichen Verluste, die Doppelstrategie - Bomben und Brot - sowie die Möglichkeit neuen Terrors beschrieben - alles gerichtet auf eine positive Zukunft: „Unsere Kinder sollen nicht in Angst aufwachsen.“ Auf der Seite gegenüber ist Osama bin Laden mit „seiner Hassrede“ abgedruckt, in der er den heiligen Krieg erklärte. „Die Amerikaner werden nie wieder in Sicherheit leben können.“ „Der heilige Krieg gegen Juden und Christen hat begonnen.“ Irrationalität und Unberechenbarkeit auf Seiten der Gegner manifestieren sich in „Hassreden“ und Gottesgeschenken: Der Extremist und der Taliban-Führer Mullah Omar haben die US-Angriffe „dank der Gnade Gottes überlebt.“ Dem gegenüber steht die Rationalität und Berechenbarkeit auf amerikanischer Seite: „Hightech-Waffen gegen Terroristen.“ Am nächsten Tag, dem 9.10.01 findet dann eine deutliche Heroisierung der Soldaten statt: „Die Nacht der Bomber-Piloten“ - 40 Kampfjets, 50 Cruise Missiles - gestern 2. Angriff." Die Bombardierung ist auf den folgenden Seiten in chronologischem Ablauf beschrieben, aber dennoch ist der Versuch, Osama bin Laden und seine Anhänger unschädlich zu machen, fehlgeschlagen: So wie Osama bin Laden und der Terrorangriff auf Amerika „aus dem Nichts“ kamen, so verschwand der Terroristenführer im „Nichts“ - in einer Höhle - „Dieser Mann hat das Höhlenbauen erfunden.“ Die „Höhle“ wirkt als ein Symbol, durch das der Westen den „Orient“ seit jeher gesehen hat, wie Edward Said in Orientalism beschreibt (Said 1981).

Die Bedrohung durch dieses „Ungreifbare“ wird für Deutschland immer konkreter: „Bald Angriffe gegen andere Länder?““ „Dieser Deutsche wollte für Bin Laden kämpfen" - darunter die Abbildung eines deutschen Jugendlichen, der sich in einem afghanischen Ausbildungslager für den Kampf schulen ließ. 
„Drohen Anschläge jetzt auch bei uns?“ Die „Viren“, die unangreifbar das System untertunneln, dringen immer weiter auch in die deutsche Innenwelt ein. Vom 10.10.01 an steht die Berichterstattung um die Milzbrandfälle, verursacht durch Anthrax in Pulverform, im Vordergrund - zuerst in Amerika. „Der Horror“, so der Titel wird flankiert von „Schröders Angebot an Präsident Bush - Deutsche Piloten nach Afghanistan“. Am 11.10.01 dann wird der Titel durch den Prozess gegen einen Kindermörder bestimmt. Die USRegierung hat unterdessen bestätigt: „Attentäter verbreiten die Todesbakterien“. Die Gefahr kommt immer näher: „Milzbrand-Alarm in Berlin“.

Am 12.10.01 wird der Ausweitung der Milzbrandfälle eine Erfolgsmeldung entgegengesetzt: „Polizei knackt Terrorzelle. Planten zwei Bin-LadenTerroristen einen furchtbaren Anschlag in Europa?“ Zeitgleich wird auf einen langen Krieg vorbereitet und das eskalierende Kriegszenario entfaltet: „Die Bombenangriffe werden wahrscheinlich noch einige Wochen anhalten." „Jetzt kommen die ,Bunker-Brecher'“" Am 13. Oktober bestimmt ein Skandal in der Unterhaltungsindustrie den Titel; das sich ausweitende MilzbrandSzenario folgt nach. Der Bericht über Bushs letzten Appell an die Taliban „Spuckt heute bin Laden aus ...“ enthält einen Kasten „Proteste gegen USA eskalieren in Pakistan“. Hier wird das Kriegsszenario weiter ausgebaut, denn Pakistan ist im Besitz einer Atombombe und eskalierende Proteste dort könnten in einen Atomkrieg führen. Vom 17.10.01 an soll offenbar nicht mehr zugelassen werden, dass der Krieg die Schlagzeilen bestimmt. Mit Ausnahme des 18.10.01, wo „Angst“ im Hinblick auf Milzbrandattentate noch einmal die Berichterstattung über die psychische Situation eines TV-Stars schlägt, gerät die Berichterstattung um den Krieg und die Folgen in die zweite Reihe. Bis zum 1.11.01 sind die Schicksale von Fernseh- und Sportstars titelbestimmend. Somit scheint die BILD-Berichterstattung sich auf eine „normalisierende“ Diskursstrategie zu verlegen: Die Banalität des Alltags gerät in den Vordergrund und damit wird alles andere in den Hintergrund gedrängt und zugleich „mit-normalisiert“ - der Krieg und die Anthraxanschläge sind zum Alltag geworden. Und so nimmt das Geschehen seinen „normalen“ Verlauf: Selbst die Meldung „EU unterstützt Boden-Truppen in Afghanistan“ bleibt am 20.10.01 zweitrangig.

Die Auswirkungen des Terrors werden verstärkt auf die Diskussion um die „innere Sicherheit“ in Deutschland übertragen. Am 22.10.01 bietet die Vergewaltigung eines 7-jährigen Mädchens in einer deutschen Schule Anlass zu der Frage: „Sind unsere Kinder denn nirgendwo sicher?“ Über der Faltkante der Titelseite ist noch zu lesen: „Bush befiehlt Geheim-Agenten ,Tötet Bin Laden“". So wird über Sachverhalte, die in keinerlei ursächlichem Zusammenhang stehen, die Verknüpfung des Terrors mit dem Thema „Innere Sicherheit“ geschaffen, mit dem Ziel, Maßnahmen zur Überwachung der Öffentlichkeit den Weg zu bereiten, die am nächsten Tag formuliert werden 
„Polizei fordert Videokameras und Wachpersonal - Macht unsere Schulen sicher." Damit wird der argumentative Kreis geschlossen, der für den Krieg sprechen soll und der oben bereits angesprochen wurde - es geht um die Sicherheit in der Zukunft, die Sicherheit „unserer Kinder“. Diese Argumentationsstrategie wird durch die titelgebenden Alltagsereignisse untermauert, die der Zufall dafür ausbeutbar machte: Die Berichte über die Hochzeit von Steffi Graf und Andre Agassi; die Geburt von Ralf Schumachers Sohn; die Frage der Frühgeburt des Kindes von Graf und Agassi; die anhaltende Trauer der TV-Moderatorin Petra Schürmann um ihre Tochter; die Geburt des Kindes von Graf und Agassi; die Diskussion des „komischen“ Names des Kindes von Graf und Agassi; das Glück der Mutter; die publikumswirksame Freizügigkeit der Mutter Jenny Elvers und - am 1.11.01 - die Ankündigung einer Hochzeit in Weiß zu Weihnachten von Steffi Graf und Andre Agassi. ${ }^{6}$ Dies ist die eindrucksvolle Liste von Ereignissen, die persönliche Schicksale von prominenten Film- und Sportstars im Hinblick auf Ehe und Familie, auf ihre Zukunft und die Zukunft ihrer Kinder darstellen. Hier wird eine Diskursstrategie deutlich, die Prominente als Applikationsvorgaben für eine positive, familienorientierte Zukunft bietet und am 1.11.01 auf Seite 1 ein Wintermärchen - die Hochzeit in Weiß zu Weihnachten in Aussicht stellt - und auf Seite 2 fragt: „Winterkrieg mit deutschen Soldaten?" „Deutsche Gefangene jetzt Geiseln der Taliban - Bundesregierung rechnet mit baldigem Einsatz in Afghanistan." Abgebildet ist „Der Schnee-Soldat“, seine Ausrüstung dezidiert beschrieben; einfach ist die Logik: Wer Wintermärchen will, muss auch Winterkriege führen.

Damit ist festgeschrieben, was am 7.11.2001 offenkundig wird, nachdem am 3.11.01 der Titel nochmals von „Milzbrand - Großalarm in Deutschland“ (der Verdacht stellte sich später als unbegründet heraus) bestimmt wurde: „Erstmals seit dem Zweiten Weltkrieg wird Deutschland Soldaten für einen Kampfeinsatz bereitstellen.“ Am nächsten Tag werden aufgrund von Äußerungen des US-Verteidigungsministers Donald Rumsfeld Zweifel laut, ob die von Kanzler Schröder präsentierten Anforderungen an die Bundeswehr im Anti-Terror-Krieg tatsächlich auf präzise Wünsche der USA zurückgehen (FR, 8.11.01, S.1). In vorauseilendem Gehorsam soll offensichtlich eine lange Entwicklung zu ihrem vorläufigen Abschluss gebracht werden: Deutschland hat das Trauma des 2. Weltkrieges überwunden und kann wieder kriegerisch aktiv werden - endlich sind wir wieder ein „normales Volk“?

6 Über die weitreichende diskursive Bedeutung von Weltstars als Applikationsvorgaben vgl. Bünger 1998.

7 Man vgl. dazu auch die sog. „Walser-Debatte“ vor dem Hintergrund der Verleihung des Friedenspreises des Deutschen Buchhandels 1998. Walser (1998) löste einen heftigen Streit über das historisch bedingte Selbstverständnis der Bundesrepublik Deutschland aus. Die Dokumentation „Endlich ein normales Volk?“ (Dietzsch u.a. 1999) zeigt die Resonanz der 


\section{Fazit}

Nach dem Ausbruch des Krieges zeigt die BILD-Berichterstattung eine Diskursstrategie, die auf langandauernde Kriegshandlungen unter Beteiligung der Deutschen vorbereitet und das eskalierende Kriegs- und Bedrohungsszenario durch Familien-Szenen aus dem Alltagsleben von Stars konterkariert und somit „normalisiert“. Die gebotenen Applikationsvorgaben sollen aufgegriffen werden unter dem Aspekt, den Bush in seiner Rede zur Rechtfertigung des Krieges nannte: „Unsere Kinder sollen nicht in Angst aufwachsen.“ Und für diese Zukunft - so wird diskursiv nahegelegt - muss Deutschland auf die außenpolitische Ebene gerichtet, das Trauma des Zweiten Weltkrieges überwinden und sich aktiv an den Kriegshandlungen beteiligen und auf innenpolitischer Ebene die Sicherheit verstärken. Dies macht bislang umstrittene Maßnahmen wie die Rasterfahndung, die Aufhebung des Bankgeheimnisses, Änderungen im Datenschutz im allgemeinen durchsetzbar und sogar „erwünscht".

Die oben gestellten Fragen nach der Berechtigung und Wirksamkeit des Krieges, der Möglichkeit, Bin Laden aufzuspüren und weitere Terroraktionen abzuwenden, werden in der BILD-Zeitung nicht breit diskutiert. Es wird lediglich auf einen wachsenden Widerstand gegen den deutschen Truppeneinsatz hingewiesen (7.11.01, S. 2) und Schröder zitiert: „Niemand ist froh über diese Entscheidung." Aber, so wird der Krieg und der Einsatz von deutschen Soldaten auf der gleichen Seite gerechtfertigt: „Dieser Krieg ist Notwehr Deutschland steht an der Seite Amerikas. Deutschland erfüllt seine Bündnispflicht. Deutschland kämpft gegen das Böse und für das Gute." Somit kann gesagt werden, dass die BILD-Berichterstattung einer Strategie der „uneingeschränkten deutschen Solidarität“ mit Amerika den Boden bereitet. Dies geschieht unter Zuhilfenahme kollektivsymbolisch aufgeladener Kodierungen des eigenen Systems, des definierten „Wir“, der „westlichen Welt“, der Bündnispartner als Innenwelt, die geschützt werden muss vor den eindringenden „Viren“ (Terroristen, Milzbrand ${ }^{8}$ ), den „Anderen“, den „Feinden“.

In dieser diskursiven Strategie ist die Gefahr einer Polarisierung angelegt, die die Welt außenpolitisch grob in eine islam-orientierte und eine nicht-islamorientierte Welt teilt und es ist zu befürchten, dass sich diese Polarisierung innenpolitisch fortsetzen wird und der Einwanderungsdiskurs mehr denn je von kulturellem Misstrauen bestimmt sein wird (vgl. FR 11.10.01, S. 1). Es

Rede in der deutschsprachigen rechtsextremen Presse auf.

8 Am 12.11.01 berichtet die Frankfurter Rundschau in Zusammenhang mit Milzbrand: FBI geht von einem Einzeltäter aus. „Der Fernsehsender CBS hat zuvor über die Annahme des FBI berichtet, dass der Absender der Anthrax-Briefe ein nicht-moslemischer US-Amerikaner sei. Eine mögliche Verbindung zum Terrornetzwerk des Moslemextremisten Osama bin Laden werde zwar nicht ausgeschlossen, doch gingen die Ermittler eher von einer einheimischen Quelle aus." (FR, 12.11.01, S. 2) 
besteht die Gefahr, dass Fremdenfeindlichkeit und Rassismus in der Gesellschaft so eine neue Grundlage finden, und Erfolge im Hinblick auf ein interkulturelles Zusammenleben gefährdet oder sogar zunichte gemacht werden. Und es besteht die Gefahr, dass dieser Rassismus noch weiter in die Institutionen unserer Gesellschaft vordringt, als es bisher der Fall ist. Somit ist zu befürchten, dass sich ein „institutioneller Rassismus“" ${ }^{\text {"9 }}$ - ein Rassismus, der in Gesetzen, Vorschriften und Institutionen angelegt ist und Menschen aufgrund ihrer Hautfarbe, Kultur oder ethnischen Herkunft diskriminiert, und welcher durch die Menschen, die für diese Institutionen arbeiten oft unbewusst oder unkritisch aufgrund ihrer diskursiven Verhaftung reproduziert wird - auf dem Nährboden dieser Diskursstrategie legitimiert und verfestigt. Diese Entwicklung kann - wie Dieter Oberndörfer in bezug auf das geplante Zuwanderungsgesetz beschreibt (Oberndörfer, in: FR 28.09.01) - an den Grundfesten einer liberalen Republik rütteln und das rückwärtsgewandte völkische Misstrauen gegen Fremde und Fremdes wieder aufleben lassen.

\section{Literatur}

Arning, Matthias: Der Terrorist sucht Aufmerksamkeit und will vor allem Angst schaffen, in: Frankfurter Rundschau, 27.09.01, S. 2

Cook, David: Die Propheten des Weltunterganges, in: Die Zeit, 20.09.01, S. 15

Bünger, Iris: „Off with her talking head - The Construction of the Subject ,Princess Diana' by British Newspapers“. In: Journal for the Study of British Cultures, Vol. 5/1, Tübingen, 1998, S. $41-54$

Dietzsch, Martin et al. (Hg.): „Endlich ein normales Volk?““ DISS, Duisburg, 1999.

Foucault, Michel: Überwachen und Strafen. Die Geburt des Gefängnisses. Frankfurt, 1977

Foucault, Michel: Wahrheit und Macht. In Ders.: Dispositive der Macht. Über Sexualität, Wissen und Wahrheit. Berlin, 1978, S. 21 - 54

Foucault, Michel: Das Subjekt und die Macht. In H.L. Dreyfus \& P. Rabinow: Michel Foucault. Jenseits von Strukturalismus und Hermeneutik. Frankfurt, 1987, S. $243-261$

Frankfurter Rundschau: Berlusconi vergleicht G-8-Gegner mit Terroristen. 26.09.01, S. 1.

Frankfurter Rundschau: Operation Dauerhafte Freiheit. 26.09.01, S. 1

Frankfurter Rundschau : USA verzichten auf Beistand der Nato. 27.09.01, S. 1

Frankfurter Rundschau: USA bombardieren Kabul. 08.10.01, S. 1

Frankfurter Rundschau: Milzbrand-Spur führt nach Iowa. 11.10.01, S. 1

Frankfurter Rundschau: Schule und Hochschule. 11.10.01, S. 1

Frankfurter Rundschau: Rumsfeld irritiert die Koalition, 8.11.01, S. 1

9 Das Konzept „institutioneller Rassismus“ wird hier in Anlehnung an den 1999 in Großbritannien veröffentlichten sog. Macpherson-Report (Macpherson 1999) verwendet, der nach dem Tod des schwarzen Jugendlichen Stephen Lawrence durch die Bestrebungen der Eltern des Opfer von der Labour-Partei initiiert wurde. Die umfassende Untersuchung erbrachte ein Fehlverhalten v.a. der Polizei in der Folgezeit des Mordes, welches eine Verurteilung der Täter unmöglich machte. Als Grundlage dieses Fehlverhaltens wird das Vorhandensein von „institutionellem Rassismus“ in der britischen Gesellschaft herausgearbeitet. Der Report fordert weitreichende legislative Veränderungen, die in ihren Auswirkungen umstritten sind. Die Übertragbarkeit dieses Konzeptes auf die Verhältnisse in der Bundesrepublik Deutschland wird auf dem Kolloquium des Duisburger Institutes für Sprach- und Sozialforschung vom 23.11.01 - 25.11.01 stattfinden, dessen Ergebnisse im Anschluss zur Veröffentlichung vorgesehen sind. 
Gerhard, Ute: Wenn Flüchtlinge und Einwanderer zu Asylantenfluten werden - zum Anteil des Mediendiskurses an rassistischen Pogromen, in Jäger, S.; Januschek, F. (Hg.): Der Diskurs des Rassismus, Oldenburg, 1992 (OBST Nr. 46), S. 163 - 178

Huhnke, Brigitta: Intermediale Abhängigkeiten bei der Inszenierung rassistischer Feindbilder seit Mitte der 80er Jahre am Beispiel der Wochenzeitungen „Bild am Sonntag“ und „Der Spiegel“, in: Jäger, S., Link, J. (Hg.): Die vierte Gewalt, Duisburg, 1993, S. 213 - 266

Jäger, Margret: BrandSätze und Schlagzeilen. Rassismus in den Medien. In: Forschungsinstitut der Friedrich-Ebert-Stiftung (Hg.): Entstehung von Fremdenfeindlichkeit. Die Verantwortung von Politik und Medien. Bonn, 1993.

Jäger, Margret: Fatale Effekte - Die Kritik am Patriarchat im Einwanderungsdiskurs, Duisburg, 1996

Jäger, Margret et al. (Hg.): Biomacht und Medien. Duisburg, 1997.

Jäger, Margret: Von deutschen Einzeltätern und ausländischen Banden - Medien und Straftaten, Duisburg, 1998

Jäger, Margret u. Jäger, Siegfried: Medien im Krieg - Der Anteil der Printmedien an der Erzeugung von Ohnmachts- und Zerrissenheitsgefühlen, Duisburg, 2001

Jäger, Siegfried: BrandSätze. Rassismus im Alltag. Duisburg, 1992, DISS. Down-load unter: http://www.uni-duisburg.de/DISS

Jäger, Siegfried: Der Großregulator: Analyse der BILD-Berichterstattung über den rassistisch motivierten Terror und die Fahndung nach der RAF im Sommer 1993. Duisburg, 1993

Jäger, Siegfried: Diskurstheorie und Diskursanalyse. Ein Überblick. In: DISS-Forschungsbericht 1995, Studien zu rechtsextremen und (neo-)konservativen Diskursen, Duisburg, 1995, S. 3-16

Jäger, Siegfried: Zur Konstituierung rassistisch verstrickter Subjekte. In: Mecheril, Paul \& Teo, Thomas (Hg.): Psychologie und Rassismus. Hamburg, 1997

Jäger, Siegfried: Kritische Diskursanalyse. 3. Auflage, Duisburg, 2001

Leontjew, A.N.: Tätigkeit, Bewusstsein, Persönlichkeit. Bonn, 1982

Link, Jürgen: Kollektivsymbolik und Mediendiskurs, kultuRRevolution 1, S. 6 - 21, 1982

Link, Jürgen: Diskursive Rutschverfahren ins vierte Reich? Rationales Rhizom, kultuRRevolution 5, 1984, S. $12-20$

Link, Jürgen: Die Analyse der symbolischen Komponenten realer Ereignisse. Ein Beitrag der Diskurstheorie zur Analyse neorassistischer Äußerungen. In: Jäger, S. \& Januschek, F. (Hg.): Der Diskurs des Rassismus, Oldenburg: OBST 46/1992, S. 37 - 52

Macpherson, Sir William: The Stephen-Lawrence-Inquiry, Cm 4262-1, London, 1999.

Oberndörfer, Dieter: Über die unerwünschten Zuwanderer. In: Frankfurter Rundschau, 28.09.01, S. 9

Said, Edward W.: Orientalism. New York, 1981. (Dt.: Orientalismus. Frankfurt/M., 1981)

The Economist: The new enemy. 15.9.2001, p. 15

The Economist: How the world has (and hasn't) changed. 27.10.2001

van Dijk, Teun: Eliten, Rassismus und die Presse in: Jäger, S.; Link, L. (Hg.): Die vierte Gewalt, Duisburg, 1993, S. 80 - 130

Walser, Martin: Erfahrungen beim Verfassen einer Sonntagsrede, Frankfurt/M., 1998 